Invest. pens. crit. (ISSN 1812-3864)

Vol. 5, No. 3, septiembre-diciembre 2017

pp. 34-58

\title{
Percepciones sobre Excelencia Educativa en las Escuelas Oficiales de Panamá
}

\author{
Dra. Nadia De León \\ Investigadora Asociada \\ INDICASAT - USMA \\ Email nadiadeleonporter@gmail.com
}

Recibido: 04 de octubre de 2017

Aceptado: 25 de octubre de 2017

\section{Resumen}

Este estudio se basa en los ensayos y entrevistas de 204 escuelas participantes en el Concurso Nacional por la Excelencia Educativa 2016, provenientes de todo Panamá, por medio de un análisis cualitativo utilizando codificación por temas, análisis de frecuencias de dichos códigos, y correlación entre ellos. Encontramos que los temas que surgen con mayor frecuencia se refieren a la infraestructura, materiales y tecnología, seguidos de actividades adicionales y concursos extra-curriculares y co-curriculares. Notamos baja correlación o ausencia de correlación entre lo que se entiende por excelencia educativa en las escuelas, lo que se hace para alcanzarla y la percepción de lo que hace falta. Se encontró también una elevada percepción de excelencia lograda y necesidad de reconocimiento a la labor llevada a cabo, así como un bajo reconocimiento de estar en una crisis educativa en cuanto a logros y calidad. La gran mayoría de las definiciones de excelencia y ejemplos de acciones tomadas hacia ella fueron poco específicos. La definición más común (48\% de las escuelas) es la educación integral, definida principalmente como aquella que incluye, aparte de lo académico o intelectual, aspectos socioemocionales (principalmente de moral) y extracurriculares. Ésta es seguida por la educación en valores (24\%). Las palabras más frecuentemente presentes en estas definiciones son: formación (15), estudiantes (17), valores (11), desarrollo (10), integral (10), centro (10), capacidades (9), académico (8), intelectual (9), aprendizaje (6), máximo (6), docentes (5), sociedad (5) y padres (5). A pesar de que aproximadamente un tercio de las escuelas incluyen a los docentes en su definición de excelencia educativa, muy pocas (por debajo del 10\%) incluyen ejemplos de esfuerzos por la mejora que tengan que ver con la preparación o el rol del docente, o el apoyo a su labor, ni tampoco notan la falta de estas actividades como barreras a la excelencia.

Palabras clave

Educación, Panamá, percepción, excelencia, calidad, prácticas. 
Invest. pens. crit. (ISSN 1812-3864)

Vol. 5, No. 3, septiembre-diciembre 2017

pp. 34-58

\section{Introducción y Justificación}

Este análisis se basa en los ensayos y entrevistas de 204 escuelas participantes en el Concurso Nacional por la Excelencia Educativa 2016, provenientes de todo el país. Las escuelas participantes presentaron ensayos de inscripción y participaron en entrevistas, en las que estaban presentes el director, un docente, un administrativo, un padre de familia y uno o varios estudiantes. Entre otras cosas, durante los ensayos y las entrevistas se les preguntaba sobre su definición de excelencia educativa y qué los hacía excelentes, ejemplos de esfuerzos llevados a cabo hacia la excelencia educativa o para mejorar la calidad de la educación, y ejemplos de qué les hacía falta para lograrlo. No se presentó una definición de excelencia ni calidad educativa, lo cual nos permite analizar los resultados para comprender, con poco sesgo externo, la manera de pensar sobre excelencia educativa en las escuelas oficiales de Panamá, qué es, cómo llegamos a ella y qué nos hace falta. Este estudio es un primer paso diagnóstico que provee información clave para informar la implementación de intervenciones en curso y diseñar futuras intervenciones que tomen en cuenta los conceptos y actitudes sobre calidad educativa y cómo lograrla presentes en el sistema, y la interacción que puedan tener con las conceptualizaciones, actitudes y prácticas inherentes a la intervención en cuestión. Entender estas percepciones es ineludible para poder realizar intervenciones efectivas con la intención de lograr la mejora continua del sistema de educación público en Panamá. Es importante notar que Esta investigación fue parcialmente financiada por la Fundación para la Promoción de la Excelencia Educativa. Su contenido es responsabilidad de la autor y no de la fundación.

\section{Antecedentes y Marco Teórico}

El consenso en ciencias de la educación es medir la excelencia educativa, o efectividad de las escuelas, con base en el aprendizaje de los estudiantes (Reynolds et al. 2001). Dicho aprendizaje suele ser medido a través de pruebas internacionales estandarizadas, ya que permiten producir información fácilmente generalizable y comparable, y se enfocan en los tipos de aprendizaje sobre cuya importancia hay común acuerdo internacionalmente.

Las investigaciones y meta-análisis de investigaciones en el campo de las ciencias de la educación identifican que la variable con mayor impacto en el aprendizaje de los estudiantes son los docentes, seguidos por los directores (Bell, Bolam, and Cubillo, 2003). La infraestructura y el uso de la tecnología tienen poco o ningún impacto en los resultados obtenidos por los estudiantes, más allá de la infraestructura básica como los baños sanitarios y salones con temperatura cómoda (OECD, 2010; OECD, 2015).

Se han generado muchísimas listas de características de escuelas efectivas, las cuales podemos resumir y agrupar, según sus resultados, de la manera siguiente: 
Invest. pens. crit. (ISSN 1812-3864)

Vol. 5, No. 3, septiembre-diciembre 2017

pp. 34-58

\section{Liderazgo}

- buen liderazgo por parte de los administradores a nivel alto y medio, con la capacidad de identificar y resolver problemas y facilitar cambios. (Smith and Tomlinson, 1990; Department of Education and Science, 1988)

- una organización y gerencia simple, clara y lo más plana posible, con participación de los docentes en procesos de auto-evaluación constantes y toma de decisiones. (Smith and Tomlinson, 1990; Brighouse and Tomlinson, 1991: 5; Sammons et al., 1995; Reynolds et al., 1996)

- objetivos claros y compartidos, traducidos a prácticas pedagógicas con énfasis en estándares académicos altos. (Department of Education and Science, 1988; Sammons et al., 1995; Reynolds et al., 1996; Reynolds,1995)

- espíritu de la escuela con un buen ambiente de trabajo y respeto, y altas expectativas para los estudiantes y el personal (Smith and Tomlinson, 1990; Rutter et al., 1979; Brighouse and Tomlinson, 1991: 5; Sammons et al., 1995; Reynolds et al., 1996)

- un ambiente positivo, ordenado y estimulante, con manejo efectivo de los estudiantes en el salón de clases (Department of Education and Science, 1988; Rutter et al. 1979; Sammons et al., 1995; Reynolds et al., 1996)

\section{Docentes}

- docentes bien capacitados con conocimiento pedagógico y de los temas que enseñan, y consistencia a lo largo de los años. (Department of Education and Science, 1988; Reynolds,1995)

- desarrollo profesional constante para el personal. (Brighouse and Tomlinson, 1991: 5)

- docentes que constituyen modelos positivos a seguir para los estudiantes (Rutter et al., 1979).

- trato y realimentación positiva a los estudiantes, con seguimiento a su bienestar integral; relaciones positivas y apoyo a su individualidad. (Smith and Tomlinson, 1990; Department of Education and Science, 1988; Rutter et al., 1979; Sammons et al., 1995; Reynolds et al., 1996)

\section{Enseñanza-Aprendizaje}

- utilización de métodos de enseñanza efectivos, estructurados y adaptables. (Sammons et al., 1995; Reynolds et al., 1996)

- un currículo bien planeado, y con consistencia entre grados y materias. (Department of Education and Science, 1988; Reynolds,1995)

\section{Comunidad}

- relaciones positivas con la comunidad e involucración de los padres. (Department of Education and Science, 1988; Brighouse and Tomlinson, 1991: 5; Sammons et al., 1995; Reynolds et al., 1996; Reynolds,1995) 
Invest. pens. crit. (ISSN 1812-3864)

Vol. 5, No. 3, septiembre-diciembre 2017

pp. $34-58$

Para el contexto especifico de países en desarrollo, se ha encontrado que los factores asociados con los logros de los estudiantes también incluyen: el tiempo de instrucción, actividad en la biblioteca de la escuela, años de formación de los docentes, y los libros y materiales educativos usados (Reynolds et al., 2000: 240). En referencia a sistemas educativos, también sabemos que en los países con éxito educativo las escuelas tienden a tener mayor autonomía (OECD, 2015).

\section{Metodología}

Además de una descripción general y análisis de frecuencia de palabras, este estudio consiste en un análisis cualitativo utilizando codificación por temas, análisis de frecuencias de dichos códigos y correlación entre los códigos.

La codificación de los ensayos y entrevistas se llevó a cabo siguiendo una teoría principalmente enraizada; es decir, no se generó un mapa conceptual de lo que se estaba buscando a priori, sino que los códigos utilizados para clasificar las ideas presentadas en los ensayos y entrevistas emergieron de los mismos textos, y el mapa conceptual se fue creando a medida que se leyeron y analizaron los textos. Sin embargo, vale la pena aclarar que esto no elimina la posibilidad de sesgo causado por las ideas y la formación profesional (en educación) del investigador, quien inevitablemente leyó y analizó los textos. También se incluyeron algunos códigos relacionados a los propósitos, temas y preguntas presentadas por el concurso y el personal involucrado. Esto resultó en alrededor de 150 códigos.

Después de codificar todos los ensayos y entrevistas utilizando Atlas.ti, se procedió a crear una codificación por escuela. Es decir, se unificó el análisis de los ensayos y las entrevistas, de modo que los datos indicaran la presencia de cada código si éste se había encontrado en el ensayo y/o en la entrevista de determinada escuela.

Finalmente se crearon algunos super-códigos útiles para el análisis cualitativo y basados en: 1) los resultados del análisis preliminar llevado a cabo con las primeras 75 escuelas cuyos ensayos y entrevistas fueron analizados, 2) el marco teórico de este estudio, 3) el tipo de información que se deseaba obtener. Dichos super-códigos incluyen, por ejemplo, agrupaciones que indican si un tema se presentó como ejemplo de esfuerzo hacia la excelencia ya sea en el ensayo y/o la entrevista; si un tema se presentó como definición de excelencia, ejemplo de esfuerzo hacia la excelencia, o como falta para poder alcanzar la excelencia; o si algunos de los códigos se presentaron y otros no. Por ejemplo: "concursos: any" incluye la mención de participación en concursos ya sea en las definiciones, los ejemplos y/o las faltas; "ejemplo o acción: jornada extendida" incluye la mención de la jornada extendida en los ejemplos de por qué su escuela se considera excelente, presentados en los ensayos y/o las acciones tomadas para mejorar la calidad de la educación en la escuela relatadas durante la entrevista; mientras que "acción: solo infraestructura" indica que la infraestructura, materiales y/o tecnología fue el único tipo de esfuerzo hacia la excelencia presentado en la entrevista (es decir, no incluye las escuelas que mencionaron infraestructura junto a otro tipo de acciones, sino sólo aquellas que mencionaron únicamente acciones relacionados a la infraestructura). Esto resultó en alrededor de 200 códigos y super-códigos. 
Invest. pens. crit. (ISSN 1812-3864)

Vol. 5, No. 3, septiembre-diciembre 2017

pp. 34-58

Una vez terminado el proceso de codificación, se procedió al análisis de frecuencias utilizando dos métodos: clasificación y presentación de patrones por orden de frecuencia en general, y clasificación y presentación de patrones por orden de frecuencia por categoría. Las categorías que se utilizaron corresponden a las preguntas presentadas en las instrucciones del ensayo y las preguntas utilizadas durante la entrevista. Las categorías son: definición de excelencia, ejemplo de esfuerzo o acción hacia la excelencia, faltas para lograr la excelencia y propósito para participar en el concurso. Finalmente, se realizó un análisis cuantitativo de correlación entre los patrones encontrados.

\section{Resultados}

\section{Descripción}

La gran mayoría de los ensayos (más del 90\%) presentan un nivel de escritura muy bajo. Es decir, presentan errores de ortografía, errores de puntuación, gramática, lógica, contenido y/o poca claridad, utilización errónea de términos y datos incorrectos.

Encontramos sólo un ejemplo que explicara con detalle algún problema en particular y cómo fue diagnosticado, mencionara qué se hizo al respecto y presentará información sobre el impacto de dicha intervención. En general, las menciones de lo que han hecho para lograr la excelencia fueron muy superficiales, tanto en los ensayos como en las entrevistas. Los siguientes ejemplos ilustran esta descripción, ya que mencionan estrategias, técnicas, herramientas, métodos, modelos, debilidades y fortalezas sin especificar cuáles:

- "La puesta en práctica de estrategias didácticas innovadoras que preparan de modo eficiente y eficaz al estudiante."

- "Se procura en buena medida la formación integral de los educandos mediante el desarrollo de estrategias, técnicas y métodos acordes con la nueva tendencia y enfoques del sistema educativo."

- "Con el transcurrir de los años nuestro centro educativo ha ido buscando mejorar las debilidades y convertirlas en fortalezas para mejores resultados sabiendo que nuestros mayores testigos son los estudiantes lo cual por ellos y para ellos son los beneficios y oportunidades que se logren".

\section{Frecuencia de palabras}

También llevamos a cabo un análisis de frecuencia de palabras. A continuación, las palabras con frecuencia mayor a 200 en orden decreciente: educación, estudiante, padres, excelencia, docentes, director, concurso, comunidad, mejora, calidad, equipo, administrativo, desarrollo, aprendizaje, valores, formación, mejor, compromiso, académico, clima, personal, capacidades, social, oportunidad, integral, cultural, MEDUCA, sociedad, recurso, enseñanza, habilidades, conocimientos, vida, profesionales, aula, logro, comprensión, infraestructura, tecnología. 
Invest. pens. crit. (ISSN 1812-3864)

Vol. 5, No. 3, septiembre-diciembre 2017

pp. $34-58$

\section{Correlaciones entre códigos}

El 50\% de las veces que se mencionó la importancia del acompañamiento al rol pedagógico del docente como parte de la definición de excelencia, también se mencionó la importancia de las actividades adicionales extracurriculares. Entre las escuelas que mencionaron tres o más ejemplos de acciones específicas tomadas para mejorar la calidad de la educación durante su entrevista, aproximadamente un tercio mencionó llevar a cabo actividades adicionales con sus alumnos, incluyendo actividades curriculares y extracurriculares. En los ensayos también notamos que aproximadamente un tercio de las escuelas que mencionaron actividades adicionales extracurriculares como ejemplos de excelencia, también mencionaron actividades curriculares. Para esta clasificación incluimos como actividades curriculares solo aquellas relacionadas a las tres áreas básicas del conocimiento medidas con pruebas estandarizadas como la que se utilizó para este concurso (lenguaje, matemáticas y ciencia). También notamos que las escuelas que mencionaban actividades adicionales extracurriculares como acciones específicas para mejorar la calidad educativa en la entrevista, también solían mencionar la participación en concursos, y esfuerzos para proveer buena alimentación a los estudiantes.

Una gran parte de las escuelas que definían la excelencia educativa como aprendizaje integral, también la definían como educación en valores, como ilustra esta cita: "educación integral basada principalmente en la educación en valores". Sólo hubo dos escuelas que mencionaron comunidades de aprendizaje profesional o esfuerzos similares para que los docentes compartan y aprendan unos de otros como ejemplos de por qué su escuela es excelente. Ambas también mencionaron la importancia del acompañamiento pedagógico al docente por parte del director o supervisor.

Algunas escuelas expresaban que han logrado la excelencia a pesar de las limitaciones del sistema educativo nacional, lo cual frecuentemente relacionaban con las limitaciones tecnológicas que afrontaban. Finalmente, de las escuelas que mencionaron en su ensayo la crisis en la educación panameña, sólo aproximadamente un cuarto de ellas lo hizo mencionando resultados de pruebas internacionales.

Notamos la baja correlación o ausencia de correlación entre códigos que teóricamente deberían estar altamente relacionados, como aquellos que se referían a un mismo tema en diferentes categorías (por ejemplo: definición: capacitación docente, ejemplo o acción: capacitación docente, falta: capacitación docente). Esto demuestra una falta de coherencia entre lo que se entiende por excelencia educativa en las escuelas y lo que se hace para alcanzarla. Estas relaciones se ilustran en las gráficas siguientes: 
Fig. 1. Frecuencia de Patrones: Infraestructura
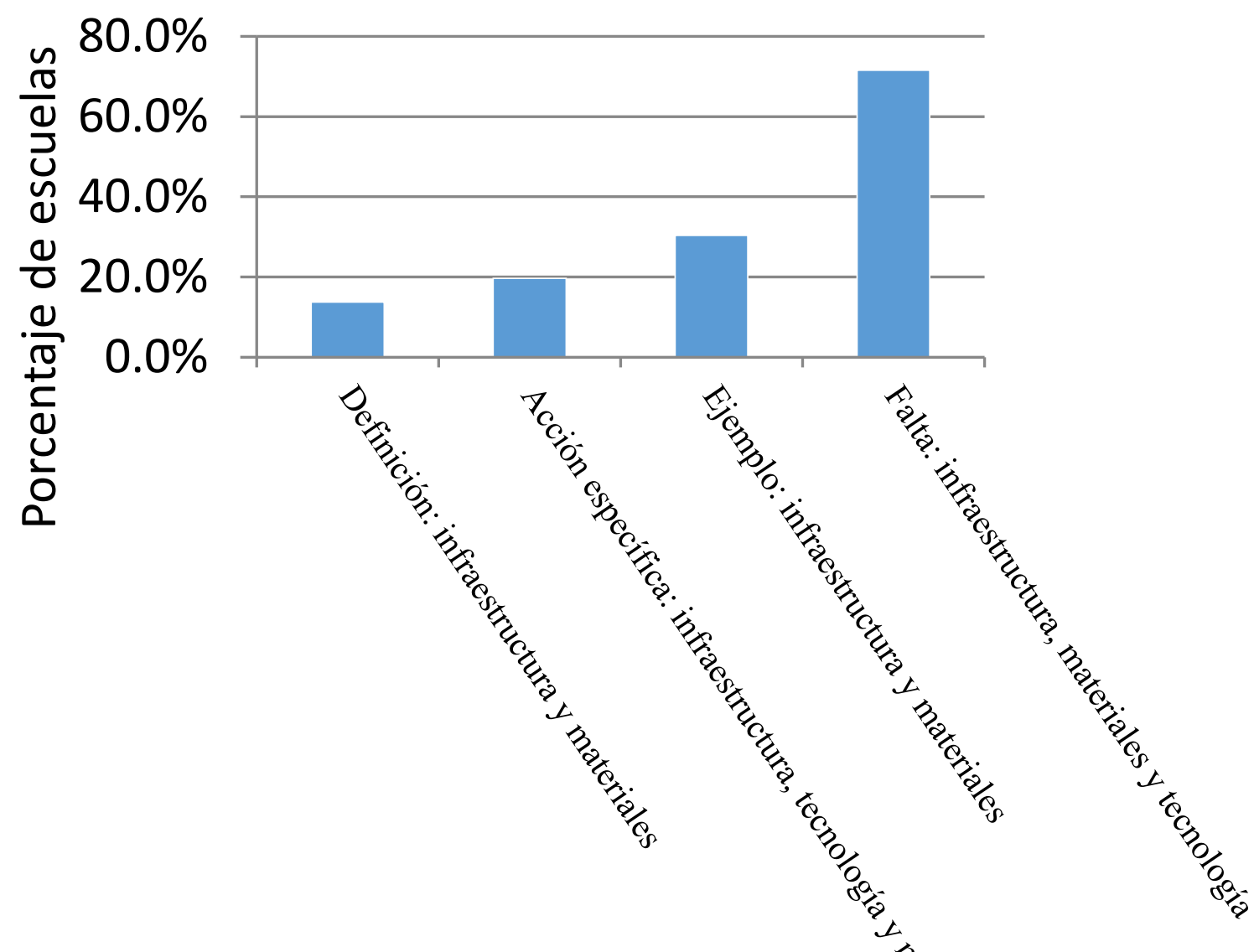
Fig. 2. Frecuencia de Patrones: Actividades Adicionales

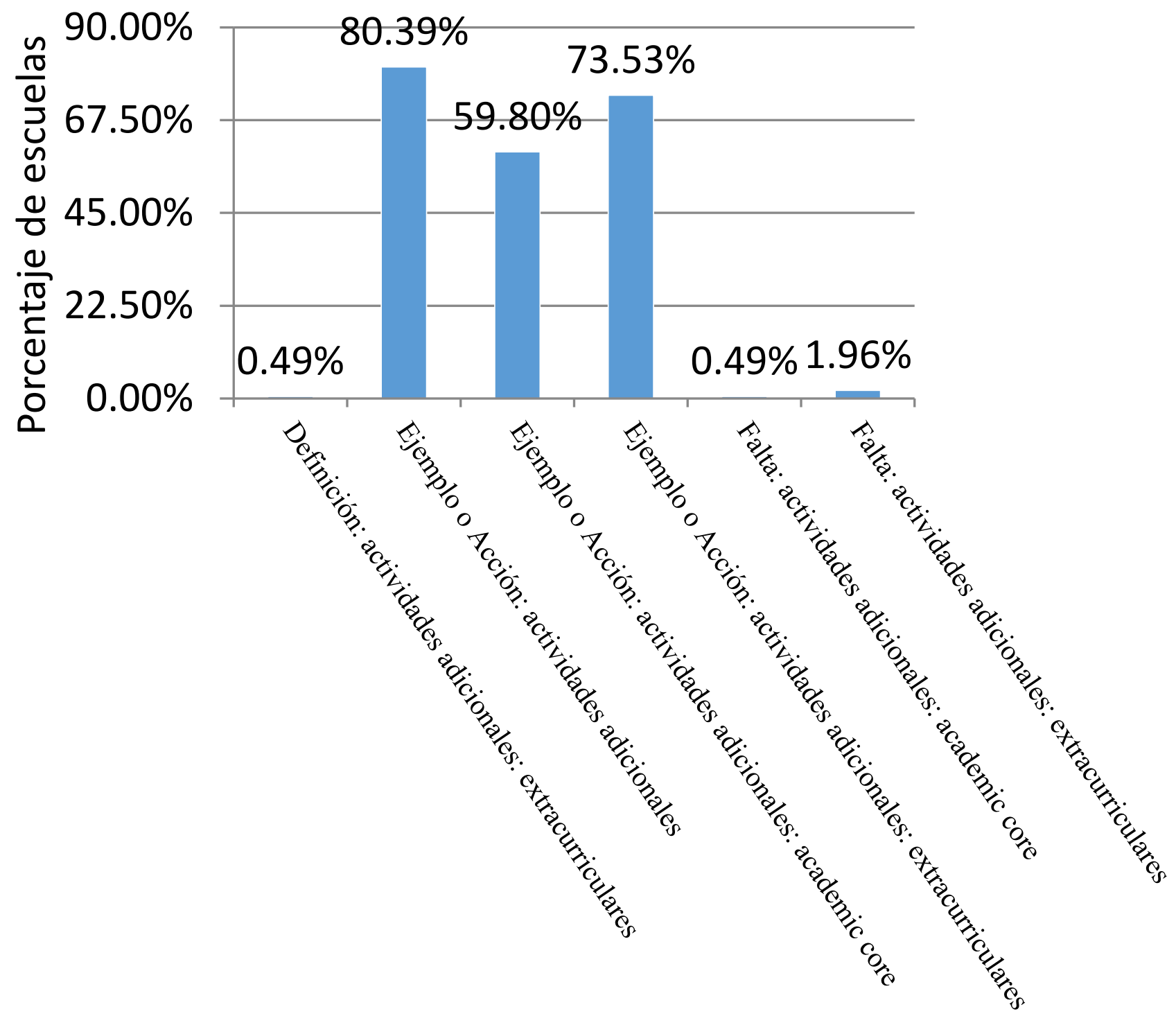


Invest. pens. crit. (ISSN 1812-3864)

Vol. 5, No. 3, septiembre-diciembre 2017

pp. 34-58

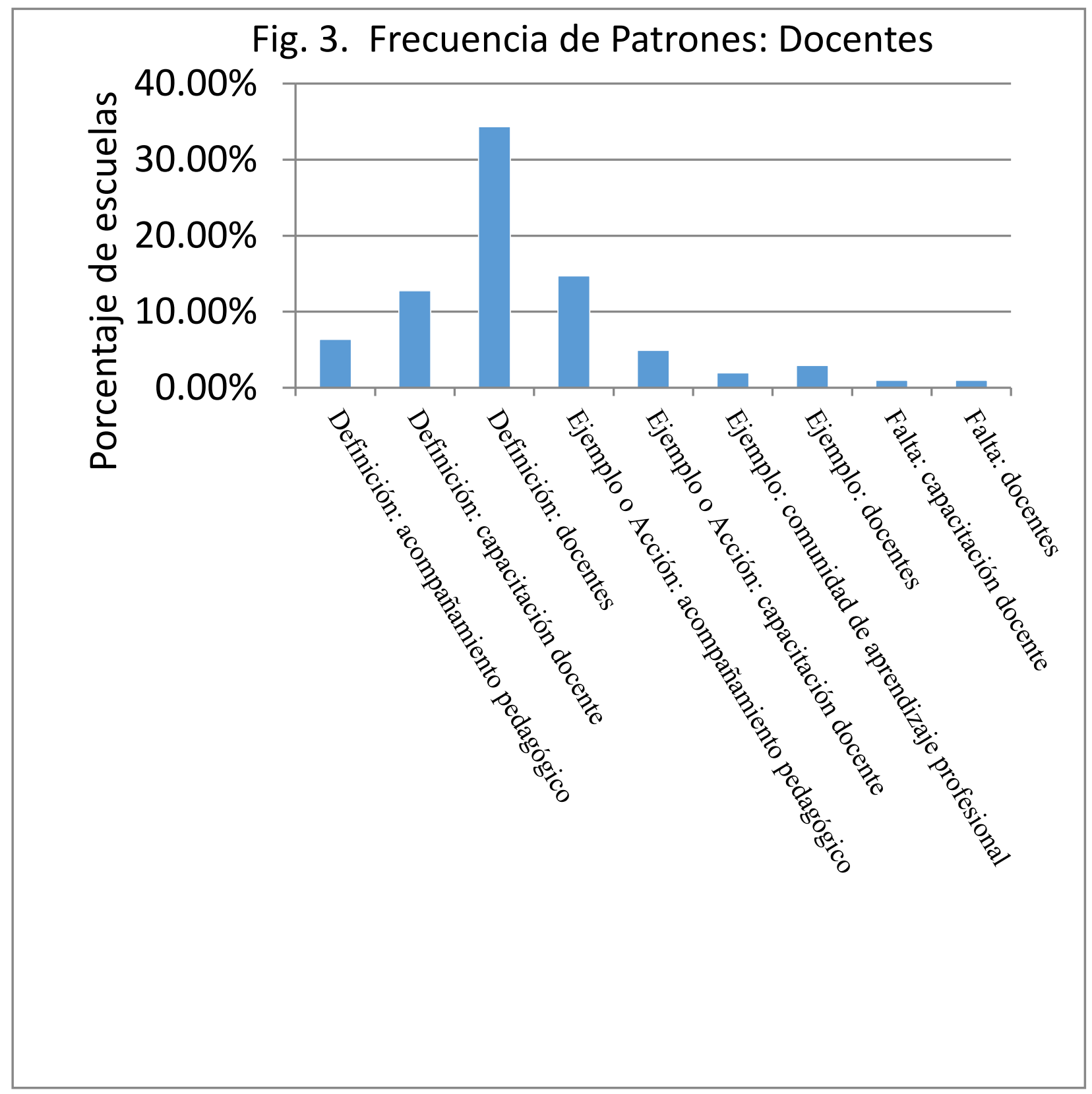

Análisis por frecuencia de patrones

Patrones con frecuencia de $80 \%$ o más

Infraestructura

El patrón que aparece con más alta frecuencia está conformado por las citas referentes a infraestructura, materiales y tecnología, mencionado por un $90 \%$ de las escuelas. Este tema emerge en 
Invest. pens. crit. (ISSN 1812-3864)

Vol. 5, No. 3, septiembre-diciembre 2017

pp. $34-58$

las definiciones y ejemplos de excelencia y esfuerzos hacia ellas, pero, es particularmente en las entrevistas cuando el tema sale más a colación, siendo el tipo de respuesta más común a la pregunta: ¿Qué le falta a este centro para alcanzar la excelencia educativa? De hecho, es el doble que el segundo tipo de respuesta (problemas en el sistema educativo, como burocracia, falta de personal, etc.) $(72 \%)$

\section{Actividades Adicionales}

El segundo patrón en orden de frecuencia son las actividades adicionales para los estudiantes, mencionados por el $80 \%$ de las escuelas. Dentro de esta categoría, incluimos actividades curriculares (olimpiadas de matemáticas, feria científica, lecto-escritura, literatura, debate), mencionadas por el 60\% de las escuelas; así como actividades extra-curriculares (Folklore, cultura, servicio social, arte y música, salud y seguridad, deportes, liderazgo), mencionadas por el 74\% de las escuelas, más actividades adicionales que no fueron presentadas con suficiente información como para clasificarlas. Es importante mencionar que aunque los porcentajes de escuelas que mencionan ambos tipos de actividades no difieren tanto entre ellos, el número de actividades mencionadas sí: en promedio los ensayos y las entrevistas mencionan 10 actividades extracurriculares por cada actividad curricular.

La participación en este tipo de actividades casi no figura como parte de las definiciones de excelencia ofrecidas en los ensayos. Sin embargo, en los ensayos, es presentada como el ejemplo de demostración de excelencia más frecuente. A su vez, durante las entrevistas, es el tipo de acción específica llevada a cabo hacia la mejora de la calidad de la educación de los alumnos presentada con más frecuencia. De hecho la frecuencia de esta categoría es más del doble en relación a la segunda de mayor frecuencia. Aunque las escuelas no definen excelencia en términos de actividades adicionales, su alto uso como ejemplo de excelencia está quizás relacionado al concepto de educación "integral” que sí está presente en la mayoría de las definiciones de excelencia de los ensayos, refiriéndose a la educación más allá de lo académico. Además, algunas citas nos explican el motivo para llevar a cabo dichas actividades como una manera de mantener a los jóvenes alejados de situaciones sociales negativas, así como una manera de motivarlos a la participación escolar y propiciar su desarrollo socio-afectivo.

\section{Patrones con frecuencia de $60 \%$ a $79 \%$}

\section{Estudiantes}

El 67\% de las escuelas mencionaron a los estudiantes. Estas citas incluyen aquellas que se refieren a que los estudiantes aprendan (60\%), aquellas que describen características presentes o deseadas en los estudiantes, etc. Muy pocas citas $(<5 \%)$ se refieren específicamente al aprendizaje de los contenidos especificados en el currículum, o a otras medidas de éxito como porcentajes de fracaso o deserción. Ninguna cita hace referencia a pruebas que midan el aprendizaje o a cómo evaluarlo.

\section{Propósito: Reconocimiento}

El 67\% de las escuelas responden a la pregunta de por qué participan en el concurso explicando que lo hacen para lograr un reconocimiento de su labor, divulgar sus logros y/o esfuerzos, y darse a conocer. De hecho, con 80 citas en total, esta categoría es tres veces más alta que el segundo propósito más común. 
Invest. pens. crit. (ISSN 1812-3864)

Vol. 5, No. 3, septiembre-diciembre 2017

pp. $34-58$

\section{Concursos}

El 60\% de las escuelas mencionan los concursos. Las citas incluyen participación en concursos cocurriculares y extra-curriculares regionales o nacionales, concursos internos, y otros reconocimientos a nivel de estudiantes o de escuela. Es importante notar la discrepancia entre el número de definiciones de excelencia que incluyen los concursos $(2 \%)$, y el número de escuelas que citan la participación en dichos concursos como ejemplos de excelencia (58\%).

Puede que la frecuencia del uso de la participación y los logros alcanzados en concursos como ejemplos de excelencia refleje la definición de excelencia como aprendizaje, y la falta de otras maneras de medir dicho aprendizaje. También es importante mencionar que encontramos siete citas adicionales que específicamente declaran participar en todos los concursos posibles como algo positivo. 
Invest. pens. crit. (ISSN 1812-3864)

Vol. 5, No. 3, septiembre-diciembre 2017

pp. $34-58$

\section{Fig. 4. Patrones con más de $60 \%$ de frecuencia}

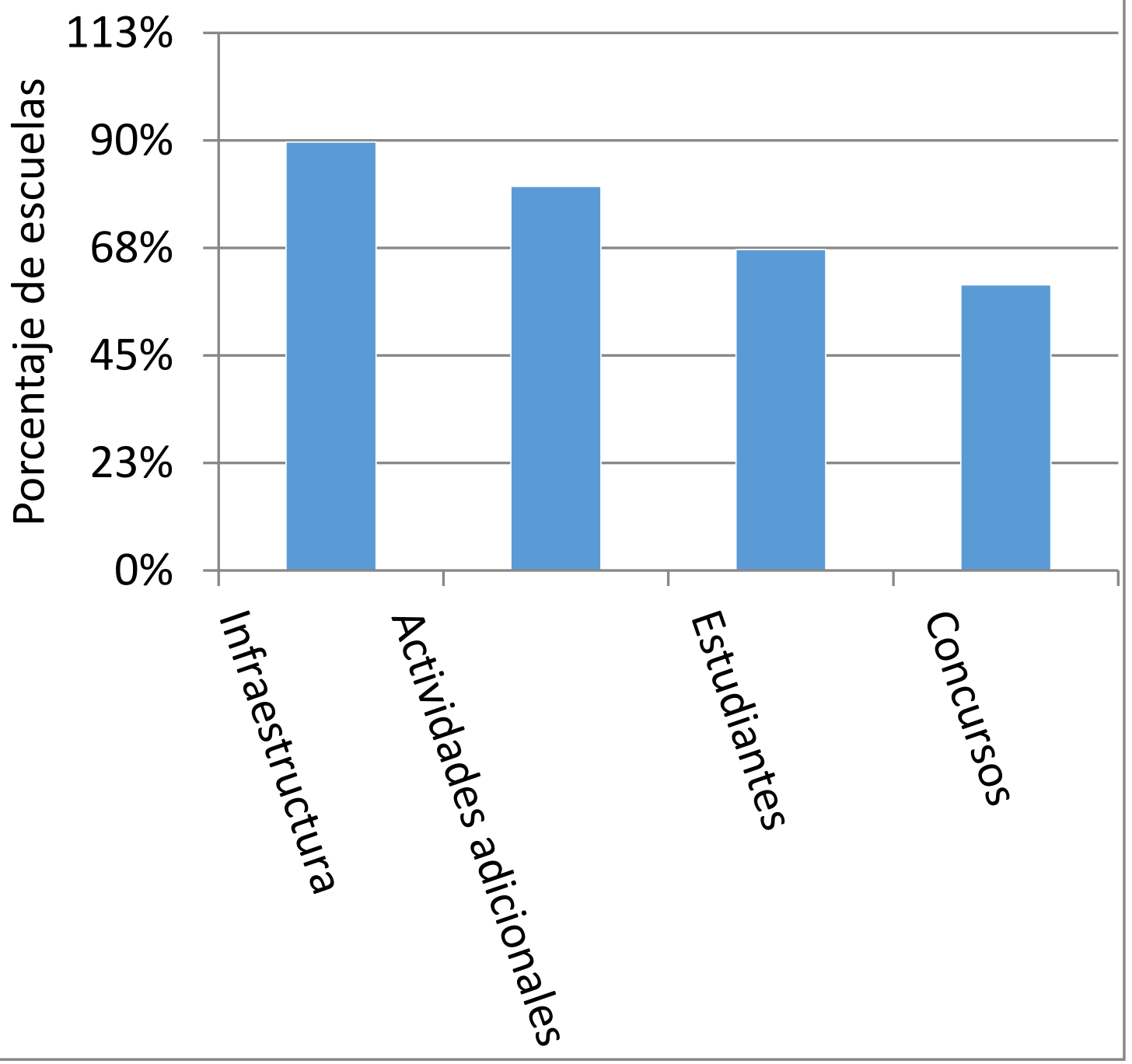

\section{Acciones específicas para la mejora}

Durante la entrevista, sólo el 62\% de las escuelas respondieron a la pregunta: ¿Recuerda alguna acción específica, de una o más personas en el centro, para lograr mejorar la calidad de la educación de los alumnos de este centro?, con al menos una acción específica. El resto mencionó ejemplos generales sin especificar la acción tomada, como por ejemplo: "La formación de líderes que aportan una valiosa ayuda a sus compañeros" sin especificar qué hacen para formarlos. 
Invest. pens. crit. (ISSN 1812-3864)

Vol. 5, No. 3, septiembre-diciembre 2017

pp. 34-58

Patrones con frecuencia de $40 \%$ a $60 \%$

\section{Educación Integral}

El patrón más común en las definiciones de excelencia educativa provistas en los ensayos se refiere a la educación integral. La definición operante entre las escuelas al referirse a educación integral se relaciona sobre todo a una educación que va más allá de lo académico y que incluye, principalmente, la moral, así como también lo extra-curricular. Hubo una alta correlación entre escuelas que utilizaban esta definición y también incluían la educación en valores como parte de su definición de excelencia educativa, a menudo, en la misma cita. A su vez, hay una alta correlación entre la educación en valores y referencias a la formación religiosa, en su gran mayoría específicamente católica o cristiana. En total, el $48 \%$ de las escuelas se refieren a la educación integral, y el 53\% a la educación integral y/o en valores.

\section{Padres}

Otro patrón con alta frecuencia está conformado por las citas referentes a los padres de familia (48\%). Entre esas citas encontramos que el 10\% describieron la alta participación de sus padres de familia como ejemplo de la excelencia de la escuela. El 30\% de las escuelas incluyeron ejemplos de excelencia o acciones tomadas para mejorar la calidad de la educación relacionadas a los padres (sobre todo escuela de padres), y el 18\% de las escuelas mencionan la falta de participación de los padres como una barrera a la excelencia.

\section{Capacitación Docente}

El 43\% de las escuelas hace mención a la capacitación docente de una u otra manera. Es de interés notar la discrepancia entre la bajísima frecuencia con la que se menciona la capacitación docente como definición o falta (menos del 7\%), y la frecuencia dentro de acciones tomadas para mejorar o ejemplos de lo que los hace excelentes $(34 \%)$.

\section{Evaluación y Liderazgo}

Si juntamos las citas que se referían a la evaluación (de los aprendizajes de los alumnos, entre otros), a las mejoras, a los cambios a nivel institucional en la escuela, y al liderazgo (definido como diagnóstico, definición de objetivo, y gestión), encontramos que el 42\% de las escuelas tienen alguna cita en esta categoría. El 30\% de las escuelas se refieren a la evaluación específicamente de alguna manera. Entre ellas encontramos ejemplos de esfuerzos hacia excelencia (35\%), y evaluación como falta para lograr la excelencia (1\%). El 14\% de las escuelas se refieren al liderazgo específicamente. Adicionalmente, el $10 \%$ de las escuelas mencionaron mejoras institucionales como ejemplo de esfuerzos hacia la excelencia, tales como cambios en el horario escolar, manera de evaluar a los estudiantes, distribución de docentes, horario escolar, sistemas disciplinarios, gestión de alimentación de los estudiantes en la escuela, programas de bilingüismo, etc. Sin embargo, con excepción de dos ejemplos muy débiles, no explican el problema que llevó a necesitar la intervención, ni tampoco explican el impacto que la intervención tuvo. 
Invest. pens. crit. (ISSN 1812-3864)

Vol. 5, No. 3, septiembre-diciembre 2017

pp. $34-58$

Patrones con frecuencia de 20 a 39\%

\section{Sistema Educativo}

El 38\% de las escuelas se refieren a dificultades dentro del sistema educativo. Durante la entrevista, cuando se les preguntó qué les hacía falta para alcanzar la excelencia, el 35\% de las escuelas respondieron que superar problemas sistémicos. A su vez, en el ensayo, el $6 \%$ de las escuelas se refieren a haber logrado los ejemplos a los que se refieren a pesar de dificultades inherentes al sistema educativo. La mayoría de estas citas se refieren a falta de infraestructura y tecnología, personal, fondos y a la lentitud e ineficiencia burocrática. Unas pocas ( $1 \%$ o menos) se refieren a la falta de formación apropiada de docentes o de currículum apropiado.

\section{Valores}

El 37\% de las escuelas se refirieron a los valores en algún momento en su ensayo o entrevista. Hay una discrepancia notable entre el porcentaje de escuelas que menciona la importancia de los valores en la definición de excelencia (24\%), y los ejemplos de esfuerzos hacia la excelencia mencionados (menos del 10\%).

\section{Comunidad Educativa}

El 32\% de las escuelas mencionan la comunidad educativa como aquella conformada por los administrativos, docentes y estudiantes, en muchos casos también los padres, y a veces también la comunidad en general donde se encuentra ubicada la escuela. La mayoría de las citas se refieren a la responsabilidad compartida y la necesidad del trabajo en equipo.

\section{Inclusión}

El 31\% de las escuelas mencionan la inclusión, en su gran mayoría la falta de inclusión como barrera a la excelencia (25\%). Incluimos dentro de esta categoría la inclusión social (servir a estudiantes en riesgo social) y la inclusión de estudiantes con necesidades educativas especiales. De ambas categorías, la segunda es muchísimo más preponderante en las citas encontradas.

\section{Religión}

El 29\% de las escuelas mencionó la religión, sobre todo como formación religiosa o espiritual, y en su gran mayoría específicamente cristiana o católica. Estas menciones se dieron sobre todo dentro de las definiciones de excelencia y a veces como ejemplos de esfuerzos hacia la excelencia, sobre todo entre actividades adicionales.

\section{A pesar de...}

El 29\% de las escuelas incluyó frases que mencionaban haber logrado lo que han logrado a la fecha, a pesar de dificultades, ya sea del contexto social en el que viven sus alumnos (19\%), limitaciones 
Invest. pens. crit. (ISSN 1812-3864)

Vol. 5, No. 3, septiembre-diciembre 2017

pp. $34-58$

económicas (11\%), dificultades con el sistema educativo (6\%) o limitaciones tecnológicas (3\%). Dentro de las menciones de problemas sociales, algunas citaban, por estar inmersas en zonas alejadas o comarcarles, los relacionados con la malnutrición, el analfabetismo de los padres y la distancia que caminan los niños para llegar a la escuela. Otras, por estar en zonas urbanas, con problemas como pandillas, drogas, violencia, embarazos precoces, hogares disfuncionales, etc.

\section{Ambiente}

Encontramos que el 20\% de las escuelas se refieren un ambiente positivo en la escuela, sobretodo como ambiente de trabajo en equipo para el personal, aunque algunas citas también se refieren a un ambiente positivo y ameno para el aprendizaje de los estudiantes.

\section{Enseñanza Activa}

El 20\% de las escuelas se refirieron en algún momento al uso de métodos de enseñanza efectivos, sobre todo la enseñanza activa, o utilizando palabras como amena /agradable/vivencial. En pocos casos $(<1 \%)$, mencionaron el constructivismo, la enseñanza por investigación y reflexión, o la educación para la vida (1\%). Casi no hubo co-ocurrencia entre las escuelas que utilizaron el concepto como definición de excelencia y las que lo usaron como ejemplo, acción o falta.

Patrones con frecuencia de 10 a $19 \%$

\section{Egresados}

Encontramos referencias a egresados, sobre todo como ejemplos de excelencia en la mayoría de los casos, por haber llegado a la universidad o por ser profesionales o, en pocos casos, mencionando personalidades famosas.

\section{Docentes}

Encontramos sobre todo referencias a docentes comprometidos, algunas referencias a docentes con formación $(<10 \%)$, y pocos $(<5 \%$ ) ejemplos de sus logros (tales como ser seleccionados como docentes modelos dentro de un programa, ganarse becas, ser escogidos para participar en proyectos de MEDUCA, etc.).

Patrones con frecuencia de 5 a 9\%

\section{Plan de centro}


Invest. pens. crit. (ISSN 1812-3864)

Vol. 5, No. 3, septiembre-diciembre 2017

pp. $34-58$

El 8\% de las escuelas se refieren a la creación y/o implementación de un plan de centro, plan de mejora y/o reglamento interno como ejemplo de esfuerzo hacia la excelencia.

\section{Alimentación}

El 7\% de las escuelas se refieren a la alimentación de sus alumnos, sobre todo a esfuerzos para mejorarla.

\section{Falta: nada}

Durante la entrevista se les preguntó qué le hacía falta al centro para alcanzar la excelencia, y en el 7\% de las escuelas la respuesta fue: nada.

\section{Labor social}

Durante la entrevista, el 6\% de las escuelas incluyó acciones de mejora a la comunidad que las rodea, tales como alfabetización de adultos y limpieza del área, al pedirles que mencionaran acciones específicas tomadas para mejorar la calidad de la educación.

\section{Mejora constante}

El 6\% de las escuelas mencionan la mejora constante como parte de la definición de excelencia o ejemplo de esfuerzo hacia ella.

\section{Falta: aprendizaje}

El 6\% de las escuelas mencionó que para lograr la excelencia hacía falta mejorar el aprendizaje de sus estudiantes.

\section{Mejoras pedagógicas}

El 5\% de las escuelas mencionan esfuerzos de mejoras en las prácticas pedagógicas del centro como ejemplo de esfuerzos hacia la excelencia, tales como agrupar a estudiantes en conjuntos multinivel para el apoyo mutuo, jornada pedagógica para la elaboración de material didáctico e implementar tutorías personalizadas con los docentes, los días viernes.

\section{Reconocimiento de crisis educativa}

El 5\% de las escuelas reconocen la crisis educativa en Panamá, y sólo el 2\% lo hace mencionando los resultados de pruebas internacionales. 
Invest. pens. crit. (ISSN 1812-3864)

Vol. 5, No. 3, septiembre-diciembre 2017

pp. 34-58

\section{Patrones con frecuencia menor al 5\%:}

\section{Acción: sólo Infraestructura}

Durante la entrevista, el 4\% de las escuelas sólo mencionó esfuerzos relacionados a la infraestructura cuando se les preguntó por acciones tomadas para mejorar la calidad de la educación de sus estudiantes.

\section{Acompañamiento pedagógico}

Sólo el 4\% de las escuelas mencionan el acompañamiento pedagógico al docente, guiando su labor.

\section{Mercado laboral}

El 4\% de las escuelas mencionaron el mercado laborar en sus ensayos u entrevistas, sobre todo al referirse a la importancia de preparar a los estudiantes para las necesidades del mercado.

\section{Análisis por Pregunta}

\section{Definición de excelencia}

La mayoría de los ensayos no incluían una definición explícita y precisa de lo que los autores consideraban como excelencia educativa. De entre las definiciones encontradas, presentamos a continuación algunos ejemplos:

- "Para nosotros, la excelencia educativa consiste en lograr que los estudiantes de nuestro plantel, a partir de sus capacidades y aptitudes, mejoren no sólo en su formación académica sino en el desarrollo de competencias que les permitan integrarse con éxito al entorno en el que viven; es sembrar en ellos la curiosidad para investigar todo aquello que no saben y que quieren saber, de resolver problemas en su diario vivir y dotarlos de los esenciales mínimos que les garantizarán su inserción, de forma exitosa, en la sociedad."

- "La excelencia educativa es para el centro educativo la llave del progreso y desarrollo de todo aquél que decide formar parte del mundo maravilloso de educar, enseñar democrática; es enseñar con amor, dedicación y, sobre todo, practicar una educación en valores. Es formar y desarrollar las capacidades de los alumnos, enseñarles a tomar decisiones, a que sean críticos, a que se amen, se respeten y valoren; significa valorarlos, oportunidad de crear un ambiente familiar, dispuesto a trabajar en equipo por el desarrollo de diversos proyectos que se planifiquen y ejecuten con eficiencia y eficacia y estableciéndose las buenas relaciones humanas permitiendo una comunicación eficaz, con acuerdos que garantizan el bienestar y potencialidad de los estudiantes." 
Invest. pens. crit. (ISSN 1812-3864)

Vol. 5, No. 3, septiembre-diciembre 2017

pp. $34-58$

- "La excelencia educativa promueve una educación integral, que atiende todas las facetas de la persona, basada en una sólida formación académica, humana y cristiana."

Se encontraron, mayormente, textos extensos, densos, difíciles de seguir, con poca claridad, que enumeraban muchas variables que los autores consideran como importantes para poder alcanzar la excelencia educativa, pero sin llegar a definir en qué consisten o señalar indicadores para reconocer cuándo se alcanzan. Estos textos fueron igualmente analizados cuidadosamente para obtener los datos aquí vertidos en referencia a la definición presentada, aunque a menudo fuese de manera implícita y no explicita.

Las palabras más frecuentemente presentes en estas definiciones incluyen formación y formar (15), estudiantes y alumno (17), valores y moral (11), desarrollo (10), integral (10), centro (10), capacidades (9), académico (8), intelectual (9), aprendizaje (6), máximo (6), docentes (5), sociedad (5), y padres (5). La definición más común (48\% de las escuelas) es la educación integral, definida a veces como aquella que incluye competencias y habilidades metacognitivas, pero principalmente como aquella que incluye, aparte de lo académico o intelectual, aspectos socioemocionales (principalmente de moral) y extracurriculares. Ésta es seguida por la educación en valores (24\%). Al agrupar las definiciones que hacen referencia a los estudiantes de al menos una manera, las encontramos presentes en un $21 \%$ de las escuelas. Estas definiciones incluyes aquellas que hacen referencia al aprendizaje de los estudiantes (14\%), al desarrollo de las cualidades socioafectivas de los estudiantes (3\%), a la participación de los estudiantes en concursos (3\%), al aprendizaje, específicamente académico o curricular (1.5\%), y a estudiantes felices (1\%). El 14\% de las escuelas se refieren a infraestructura, tecnología o materiales en su definición de excelencia; el 13\% a los docentes y el 11\% a un ambiente positivo, ya sea para el personal que labora en la escuela y/o los alumnos. Solamente entre el 5 y el 10\% de las escuelas mencionaron en sus definiciones lo siguiente: conceptos de mejora constante; uso de métodos de enseñanza activos, constructivos u otros relacionados; capacitación docente. Menos del 5\% mencionaron la necesidad de la evaluación para la mejora, se refirieron al currículum o al acompañamiento al rol pedagógico de los docentes. 
Invest. pens. crit. (ISSN 1812-3864)

Vol. 5, No. 3, septiembre-diciembre 2017

pp. 34-58

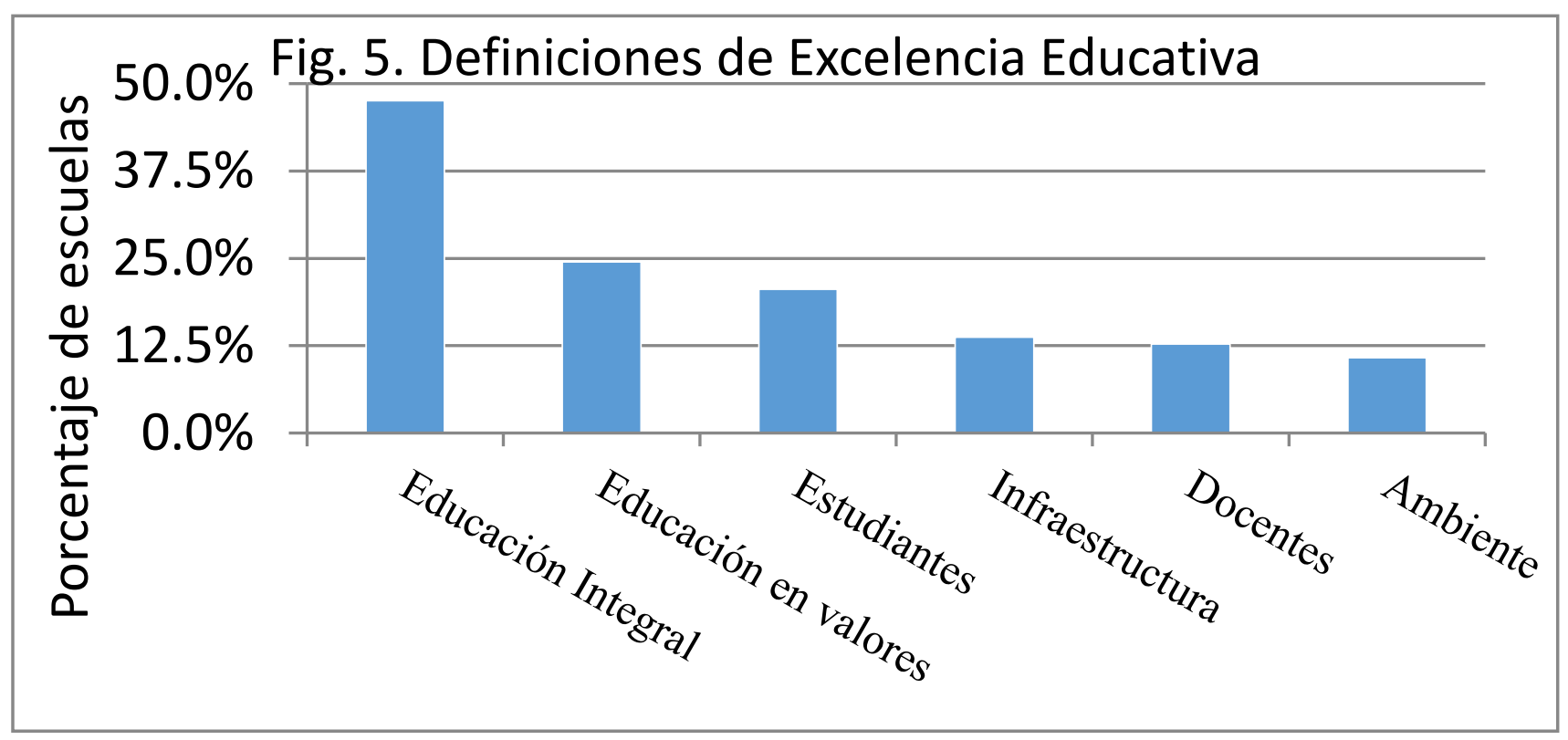

Notamos muchos ensayos y entrevistas que explicaban la excelencia de la escuela basándose en la historia de la misma, el número de estudiantes, el crecimiento de la escuela, la inclusión, o la localización geográfica del centro. Muchas citas ilustran conceptos de excelencia no relacionados a los logros académicos ("la excelencia de nuestro centro educativo no consiste en mostrar los más altos porcentajes académicos, ni mostrar la más altas calificaciones"), enfocados a la tecnología ("La excelencia de un centro educativo consiste en tener todo lo moderno al servicio del estudiante, de los maestros, administrativos, de la comunidad en general"), y la infraestructura, o basados meramente en el esfuerzo y el ambiente proporcionado y no en lo mediblemente alcanzado ("La excelencia educativa es una expresión lingüística que define claramente la vida misma del centro escolar, parte por el grado de responsabilidad, compromiso y mística que demuestra permanentemente el cuerpo docente, los administrativos y padres de familia").

Ejemplos de excelencia o acciones hacia la excelencia.

Muchos ejemplos eran muy poco específicos, como en el caso de esta cita:

"Entre algunas acciones del pasado que demuestran el compromiso del centro educativo Sector Sur con la excelencia educativa se pueden mencionar: enseñanza aprendizaje, intereses de la población a quienes van dirigidos, reforzar los contenidos académicos."

El ejemplo más común son las actividades extracurriculares para estudiantes (74\%); seguido de las actividades co-curriculares para estudiantes $(60 \%)$ y los concursos $(58 \%)$; uso de o mejoras a la infraestructura, materiales o tecnología (42\%); la capacitación docente (34\%), esfuerzos relacionados a los padres (30\%), ejemplos de liderazgo o mejoras a nivel institucional (24\%), esfuerzos relacionados 
Invest. pens. crit. (ISSN 1812-3864)

Vol. 5, No. 3, septiembre-diciembre 2017

pp. $34-58$

a la inclusión (17\%), ejemplos de docentes $(15 \%)$, ejemplos relacionados a la educación en valores $(10 \%)$, evaluación (8\%), planes de centro (8\%), ambiente en la escuela $(7 \%)$, labor social (6\%), y mejoras pedagógicas (5\%). Los ejemplos y acciones mencionadas se presentan en la tabla siguiente:

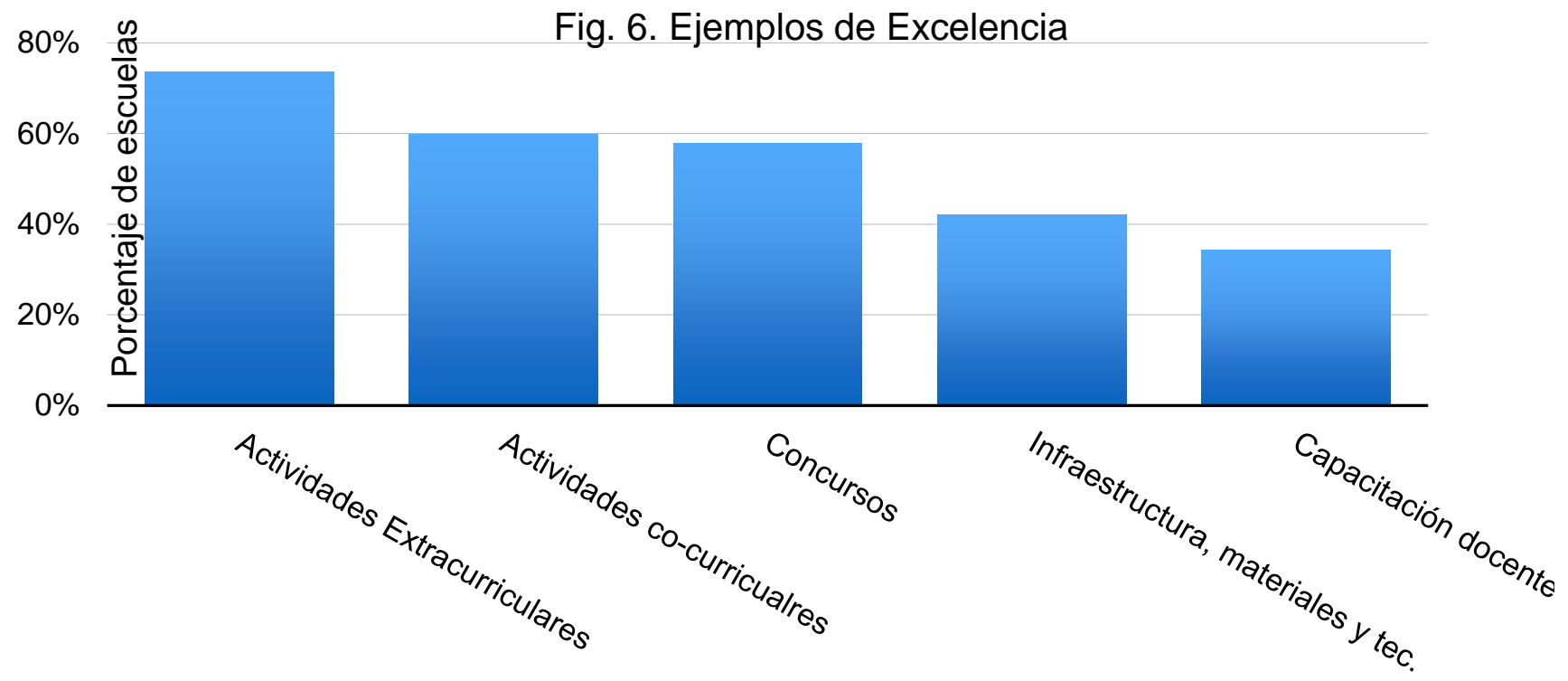

\section{Lo que falta para poder alcanzar la excelencia.}

La categoría de respuesta más común es aquella que incluye referencias a infraestructura, materiales y tecnología $(72 \%)$, seguida de dificultades en el sistema educativo, como demasiada burocracia y falta de personal $(35 \%)$, apoyo de los padres $(18 \%)$ e inclusión de estudiantes con necesidades especiales $(13 \%)$, motivación de los estudiantes $(7 \%)$, valores $(7 \%)$, las respuestas que indicaban que no hacía falta nada $(7 \%)$, aquellos que indicaban que hacía falta mejorar el aprendizaje de los estudiantes (6\%), y aquellos que indicaban la falta de capacitación docente (5\%). Todas las otras categorías de respuestas se presentaron en menos del 5\% de las escuelas. 
Invest. pens. crit. (ISSN 1812-3864)

Vol. 5, No. 3, septiembre-diciembre 2017

pp. 34-58

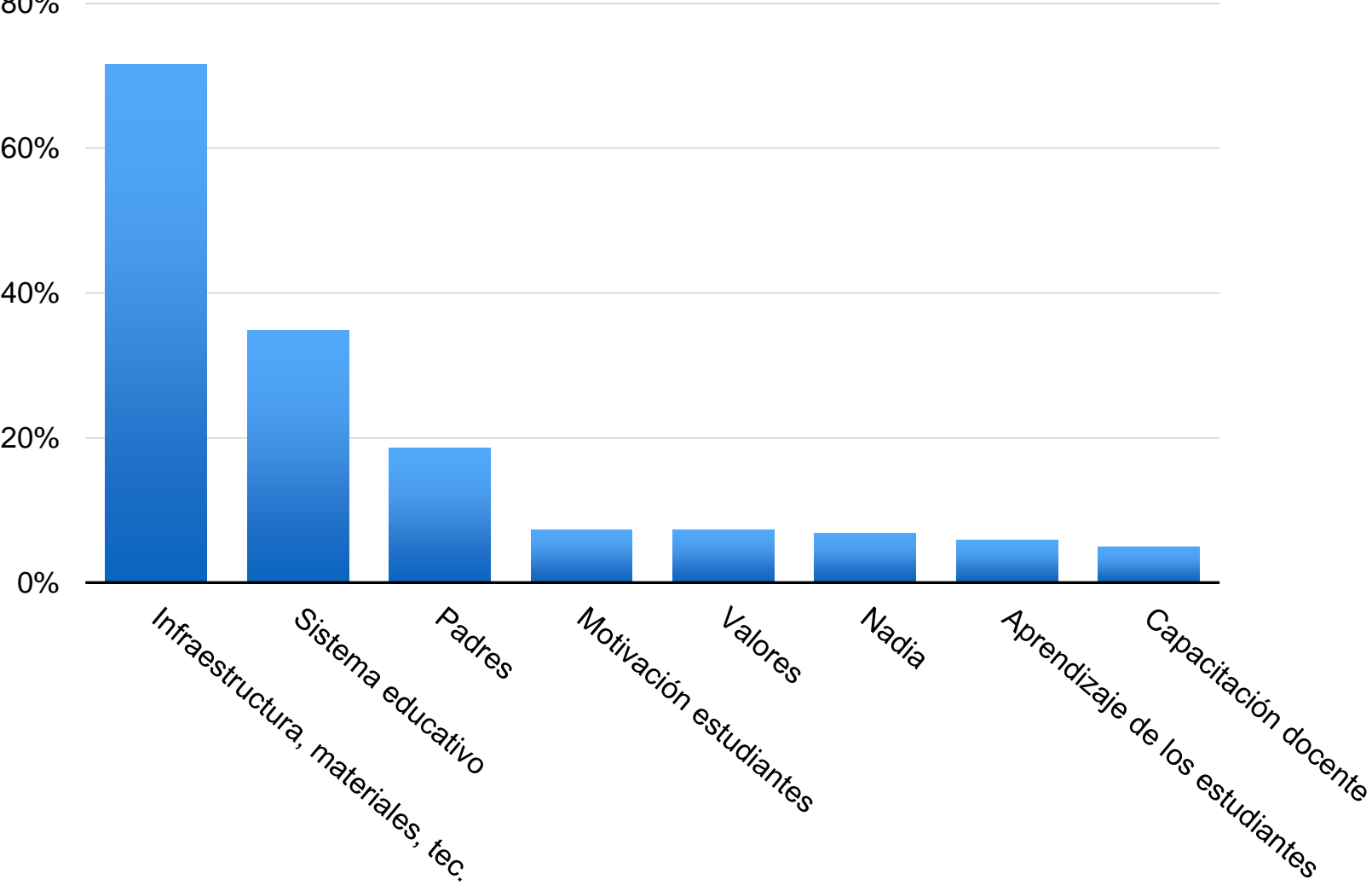

\section{Discusión}

\section{Docentes, procesos de enseñanza-aprendizaje, y educación integral y en valores.}

A pesar de que aproximadamente un tercio de las escuelas incluyen a los docentes en su definición de excelencia educativa, muy pocos (por debajo del 10\%) incluye ejemplos de acciones que tengan que ver con la preparación o el rol del docente, o el apoyo a su labor, ni tampoco notan la falta de estas actividades como barreras a la excelencia. Resulta de interés la bajísima proporción de escuelas que mencionan la falta de capacitación docente como una barrera a la excelencia $(7 \%)$.

Preocupa también el bajísimo nivel de ejemplos y definiciones relacionadas a la utilización de pedagogías efectivas por parte de los docentes, y la total ausencia de respuestas por partes de las escuelas que reconozcan que la mayoría de los docentes no utilizan estos métodos efectivamente como algo que les falta para llegar a la excelencia. Estos resultados comprueban lo indicado por los estudios en el tema: el factor más importante en la excelencia educativa son los docentes y la calidad de trabajo, que sólo puede ser proporcional a su formación inicial y continúa.

Llama la atención la baja proporción de definiciones de excelencia relacionadas al aprendizaje de los estudiantes (menos de un tercio), sobre todo en lo que se refiere específicamente al aprendizaje 
Invest. pens. crit. (ISSN 1812-3864)

Vol. 5, No. 3, septiembre-diciembre 2017

pp. $34-58$

curricular, que es, al fin y al cabo, el propósito final de la educación y, por lo tanto, la única medida válida de su éxito. Quizás a eso se deba que la participación y logros en concursos aparezcan tan frecuentemente como ejemplo de excelencia (presente en prácticamente todas las escuelas). Esto es a pesar de que muchos de estos concursos son sobre temas extra-curriculares o, aunque sean sobre conocimientos curriculares, no están medidos de manera científicamente valida y confiable. Hay poca conciencia de deficiencias en el aprendizaje de nuestros estudiantes, tanto a nivel nacional como a nivel de escuela, casi ninguna referencia a estándares internacionales, pocas referencias al concepto de mejora continua, y una gran parte de las escuelas que participaron expresan ya haber alcanzado la excelencia y estar participando para poder ser reconocidos por ello. Sólo una de las escuelas mencionó de alguna manera la falta de evaluación como barrera a la excelencia.

Entre nuestras escuelas, la principal definición de excelencia parece consistir en un concepto superficial de educación integral, altamente basado en valores y otros aspectos socioemocionales, aunque también menciona habilidades metacognitivas, pero que no se concatena con los esfuerzos realizados en las escuelas hacia la excelencia. Esta manera de concebir la excelencia educativa, en gran parte como proceso de socialización y no sólo de formación intelectual, puede estar presentando una barrera filosófica entre los líderes de las escuelas y aquellos que enfatizan los conocimientos académicos básicos y su evaluación cuantificada. De la misma manera, es de interés la poca concordancia entre definiciones de excelencia, esfuerzos hacia ella y conciencia de lo que falta para lograrla; aumentada por la falta de concordancia entre las definiciones de excelencia presentes en las escuelas y los esfuerzos hacia ella, y las definiciones que se manejan a nivel internacional y los esfuerzos efectivos hacia ella identificados en la literatura de ciencias de la educación.

Fig. 7. Conciencia de deficiencias

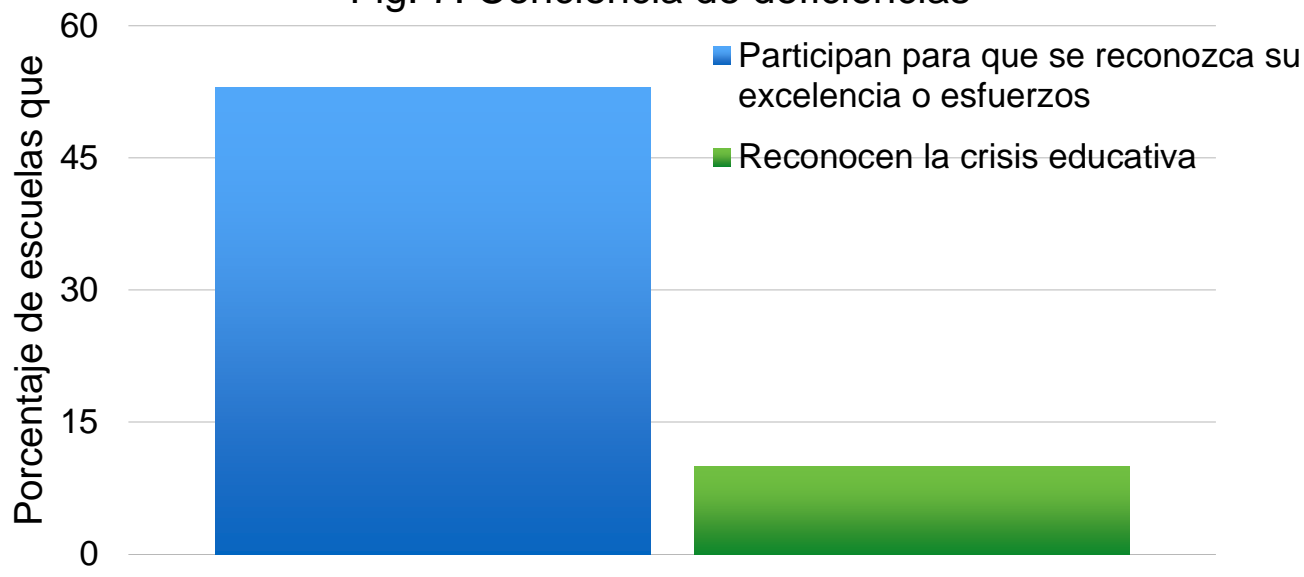

\section{Directores, acompañamiento pedagógico, liderazgo y evaluación.}

Tomando en consideración el alto impacto del rol del director en la calidad educativa, llama la atención la falta de evidencia de evaluación y liderazgo en los ensayos y entrevistas, así como la superficialidad de los ejemplos mencionados que demuestran poca capacidad de utilizar evidencias, diagnosticar dificultades, o medir éxito utilizando indicadores; aparte del bajo nivel de los ensayos escritos, muchos de los cuales fueron redactados o revisados por los directores de los centros. 
Invest. pens. crit. (ISSN 1812-3864)

Vol. 5, No. 3, septiembre-diciembre 2017

pp. 34-58

\section{Los patrones más comunes no son los de mayor impacto positivo en la calidad de la educación.}

Vemos que los principales ejemplos de barreras a la excelencia presentados por nuestras escuelas son, por mucho, la infraestructura, materiales, o tecnología, y las actividades adicionales para estudiantes (presente en prácticamente todas las escuelas, y refiriéndose en la mayoría de los casos específicamente a actividades extra-curriculares y no a aquellas relacionadas a lenguaje, ciencias y matemáticas). Esto es así a pesar de que ninguno de los dos temas figura prominentemente en su definición de excelencia educativa, demostrando, otra vez, una falta de coherencia entre ideas y acciones.

Resalta, entonces, la cantidad de tiempo y atención dedicada a actividades adicionales extracurriculares y concursos por parte de estudiantes, docentes y directivos; así como la cantidad de tiempo y atención dedicada por parte de los directores a la gestión relacionada a la infraestructura, dadas las deficiencias que presentan nuestros estudiantes en conocimientos académicos básicos. Dada que las investigaciones en ciencias de la educación han demostrado el bajo impacto de la infraestructura y tecnología en la calidad de la educación, es lamentable que sea el patrón más alto encontrado en los ensayos y entrevistas. Sin embargo, notamos que este patrón se comporta de manera especial en Latinoamérica, donde a veces no se logran las necesidades de infraestructura básicas en las escuelas, como baños, agua corriente y una temperatura adecuada, lo cual sí tiene un impacto negativo en los aprendizajes de los estudiantes. Una posible explicación para el sobre-énfasis en infraestructura, entonces, puede deberse a un fenómeno organizacional generado por una historia de falta de infraestructura básica y la continuación de dicho problema, todavía hoy en día, en algunas áreas y escuelas del país.

\section{Otros patrones de interés.}

Aproximadamente la mitad de las escuelas demostraron preocupación y esfuerzos por el rol de los padres en la educación de sus hijos. De hecho, la palabra padres o acudientes aparece con mayor frecuencia que la palabra docentes.

Además, encontramos que en las escuelas hay: 1) mucha preocupación por la inclusión de estudiantes con necesidades educativas especiales; 2) mucho énfasis en los retos que el contexto social y las limitaciones económicas presentan a las escuelas; 3) una fuerte percepción de que problemas con el sistema educativo (como la burocracia y la falta de personal) son una barrera clave para llegar a la excelencia educativa, y 4) una concepción de excelencia educativa que incluye, preocupación por, y acciones en función a la formación religiosa, en su mayor parte específicamente cristiana, de los estudiantes. Estos cuatro temas surgieron en los ensayos y/o entrevistas de aproximadamente un tercio de las escuelas.

\section{Necesidad de Reconocimiento.}

El hecho de que la mayoría de las escuelas (aproximadamente dos tercios) hayan participado en el concurso para lograr un reconocimiento de la labor que están llevando a cabo y que consideran ya excelente, refuerza el bajo nivel de conciencia sobre las deficiencias que enfrenta nuestro sistema 
Invest. pens. crit. (ISSN 1812-3864)

Vol. 5, No. 3, septiembre-diciembre 2017

pp. $34-58$

educativo, y a la vez demuestra que nuestras escuelas públicas se hayan necesitadas de reconocimiento por su ardua labor, quizás otra razón por la que participan en tantos concursos.

\section{Recomendaciones}

Los resultados de esta investigación nos sugieren como formas efectivas de ayudar a mejorar la calidad de la educación en Panamá, o al menos en estas escuelas, las siguientes:

- Promover la comprensión por parte de directores, docentes y administradores públicos, de la baja correlación existente entre infraestructura, materiales y tecnología, y logros en aprendizajes académicos básicos de los estudiantes, demostrada por estudios a la fecha en ciencias de la educación.

- Promover la comprensión por parte de directores, docentes y administradores públicos, de la importancia de la capacitación docente continua y el acompañamiento pedagógico al docente.

- Crear conciencia sobre la falta de capacitación docente y acompañamiento pedagógico de alta calidad.

- Promover la comprensión por parte de directores, docentes y administradores públicos, de la importancia de la evaluación de los aprendizajes de los estudiantes.

- Crear conciencia sobre la falta de evaluación confiable de los aprendizajes de los estudiantes.

- Juntar a actores con diferentes concepciones sobre la excelencia educativa, para lograr consenso y facilitar el trabajo en equipo en el futuro.

Los resultados de esta investigación sugieren la importancia de estudiar a mayor profundidad:

- La existencia de un concepto de excelencia no universal sino dependiente del contexto de cada escuela, en el cual las expectativas para los estudiantes de ciertas escuelas del país sean menores que las expectativas para otros.

- Las posibles barreras generadas por una definición de excelencia educativa altamente basada en lo no-académico.

- La realidad de los estudiantes clasificados como con Necesidades Educativas Especiales.

- El tipo de actividades adicionales, curriculares y extra-curriculares, y concursos en los que se involucran los estudiantes, así como las capacitaciones en las que participan los docentes, para entender las características de las opciones presentes y su uso, así como evaluar confiablemente su efectividad por medio del impacto en las acciones de los docentes y el aprendizaje de los estudiantes. 
Invest. pens. crit. (ISSN 1812-3864)

Vol. 5, No. 3, septiembre-diciembre 2017

pp. 34-58

\section{Referencias}

Bell L, Bolam R, Cubillo L (2003) A systematic review of the impact of school leadership and management on student outcomes. In: Research Evidence in Education Library. London: EPPICentre, Social Science Research Unit, Institute of Education, University of London.

Brighouse, T. and Tomlinson, J. (1991) Successful Schools. London: Institute of Public Policy Research.

Department for Education and Employment (DfEE) (2000), National Standards for Headteachers. London: DfEE.

OECD (2015), Students, Computers and Learning: Making the Connection, OECD Publishing, Paris.

OECD (2013), PISA 2012 Results: What Makes Schools Successful. OECD Publishing, Paris

OECD (2010), PISA 2009 Results: What Makes Schools Successful. OECD Publishing, Paris

Reynolds, D. (1991) School effectiveness in secondary schools: research and its policy implications. In S. Riddell and S. Brown (eds) School Effectiveness Research: Its Messages for School Improvement. Edinburgh: HMSO.

Reynolds, D. (1995) The effective school: an inaugural lecture. Evaluation and Research in Education, 9 (2), 57-73.

Reynolds, D. (2000) School effectiveness: the international dimension. In C. Teddlie and D.

Reynolds (eds) The International Handbook of School Effectiveness Research. London: RoutledgeFalmer.

Reynolds, D., Sammons, P., Stoll, L., Barber, M. and Hillman, J. (1996) School effectiveness and school improvement in the United Kingdom. School Effectiveness and School Improvement, 7 (2), 133-58.

Reynolds, D., Teddlie, C., Creemers, B., Scheerens, J. and Townsend, T. (2000) An introduction to school effectiveness research. In C. Teddlie and D. Reynolds (eds) The International Handbook of School Effectiveness Research. London: RoutledgeFalmer.

Reynolds, D., Hopkins, D., Potter, D., and Chapman, C. (2001) School Improvement for Schools Facing Challenging Circumstances. London: DES.

Sammons, P. (1994) Findings from school effectiveness research: some implications for improving the quality of schools. In P. Ribbins and E. Burridge (eds), op. cit.

Sammons, P., Hillman, J. and Mortimore, P. (1995) Key Characteristics of Effective Schools: a Review of School Effectiveness Research. Report by the Institute of Education, University of London, for the Office for Standards in Education. 